AMBIENT APPRAISAL

\title{
An Update on the Global Prediction of Thermohydrogravidynamic Principle Concerning the Strongest Intensifications of the Seismotectonic Processes: Special Reference to California
}

\section{Sergey V. Simonenko}

V.I. Il'ichev Pacif ic Oceanological Institute, Far Eastern Branch of Russian Academy of Sciences, Vladivostok, Russia

Study Area: Vladivostok, Russia

Coordinates: $43^{\circ} \mathrm{o} 8^{\prime} \mathrm{N} ; 131^{\circ} 54^{\prime} \mathrm{E}$

Key words: Cosmic Geophysics, Cosmic Seismology, Non-stationary Cosmic Gravitation, Natural Disasters

\section{Abstract}

The prediction on the established thermo-hydrogravidynamic principle concerning the strongest intensifications of the seismotectonic and climatic processes in California (since 9 August 2017 and before 3 March 2018) was determined by the maximal combined planetary and solar integral energy gravitational influence on the internal rigid core of the Earth and on the Earth as a whole. This demonstrates that the global prediction on thermohydrogravidynamic principle (used for the planets and the Sun) could be considered as the signif icant practical contribution to environmental science. It is related with the possibility for predicting (in advance) the modern strongest regional intensifications of the seismotectonic and climatic processes of the Earth subjected to the energetic cosmic (planetary, solar and lunar) gravitational influences.

due to the gravitational interactions of the Sun with Jupiter Saturn, Uranus and Neptune (Simonenko, 2017).

By analyzing the dynamism of (as per U.S. Geological Survey) earthquakes occurred in California (since Aug. 9, 2017 and before Nov. 27, 2017), the partial prediction of the thermohydrogravidynamic theory concerning the different forthcoming ranges (characterized by the corresponding calculated probabilities) of the strongest intensif ications of the seismotectonic and climatic processes for the same was also pre-estimated. The aim of this article is to present the total dynamism analysis of the earthquakes occurred in California (since 9 Aug. 2017 and before 3 Mar. 2018) and review the relevant predictions (Simonenko, 2017) of the thermohydrogravidynamic theory concerning the strongest intensifications of the seismotectonic processes in California for the same time.

The generalized gormulation of the first law of thermodynamics and the global prediction on thermohydrogravidynamic principles:

Based on the general equation of continuum movement (Gyarmati, 1970), the classical differential formulation (de Groot \& Mazur, 1962) of the first law of thermodynamics for the one-component micro differential continuum element with no chemical reactions, the classical decomposition for the pressure tensor, the viscous-stress tensor and the symmetric stress tensor, the generalized differential 
formulation of the first law of thermodynamics was derived (Simonenko, 2007, 2009, 2012, 2013, 2014a,b). The general formulation is founded for individual finite continuum region $\tau$ considered in a Galilean frame of reference with respect to a Cartesian coordinate system $K$ (Fig.-1). Along with the classical terms (Gibbs, 1873; Landau \& Lifshitz, 1976), the general formula contains the established infinitesimal combined (cosmic \& terrestrial) nonstationary energygravitational influence

$$
\mathrm{dG}=\mathrm{dt} \iint_{\tau} \frac{\partial \psi}{\partial \mathrm{t}} \rho \mathrm{dV}
$$

on the continuum region $\tau$ during the time interval $d t$. The relation (1) for $d G$ takes into account the partial derivative $\partial \psi / \partial \mathrm{t}$ of the potential $\psi$ of the combined (cosmic and terrestrial) non-stationary gravitational field, the local mass density $\rho$ of the differential volume $d V$ in the continuum region $\tau$.

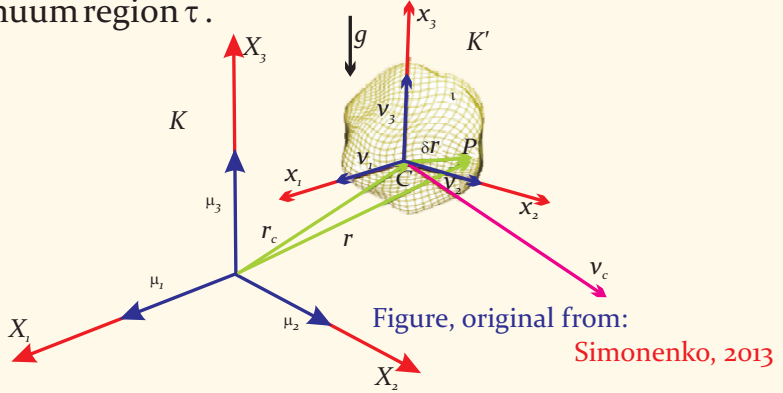

Figure-1: Cartesian coordinate system $K$ of a Galilean frame of reference and an individual finite continuum region $\tau$ subjected to the non-stationary combined (cosmic \& terrestrial) Newtonian gravitation field and non-potential terrestrial stress forces

Based on the relation (1) (used for the internal rigid core of the Earth), it was formulated (Simonenko, 2012, 2014b, 2017). The thermo-hygro-gravidynamic processes are subjected to the combined cosmic energy gravitational influence of the planets of the Solar System, the Sun (owing to the gravitational interaction of the Sun with the outer large planets) and the Moon. The global prediction thermo -hydro-gravidynamic principles (determining the maximal temporal intensifications near the time moments $t=t^{*}\left(\tau_{\mathrm{cr}}\right)$ \& $\mathrm{t}=\mathrm{t}_{*}\left(\tau_{\mathrm{cr}}\right)$ and respectively, of the thermohydrogravidynamic processes) are related with the maximal and minimal combined cosmic integral energy gravitational influences on the internal rigid core of the Earth. The global prediction on thermohydrogravidynamic principles takes into account combined cosmic integral energy gravitational influences on the considered internal rigid core (of the Earth) of the planets of the Solar System, the Sun (owing to the gravitational interaction of the Sun with the outer large planets) and the Moon. The global prediction thermohydrogravidynamic principles are formulated (Simonenko, 2012, 2014b, 2017) for the internal rigid core (of the Earth) characterized by the mass density $\rho_{c, r}=12800 \mathrm{~kg} \cdot \mathrm{m}^{-3}$ (Alboussière et al., 2010). The maximal temporal intensifications are determined by the temporal maximal and minimal combined cosmic integral energy gravitational influences (respectively, for the time moments $\left.\mathrm{t}=\mathrm{t}^{*}\left(\tau_{\mathrm{cr}}\right) \& \mathrm{t}=\mathrm{t} *\left(\tau_{\mathrm{cr}}\right)\right)$ on the considered internal rigid core (of the Earth) of the planets of the Solar System, the Sun and the Moon.

\section{Total validity concerning the predicted} strongest intensifications of the seismotectonic processes in California in 2017 \& 2018:

The forthcoming ranges of the active forthcoming intensifications of the natural (seismotectonic and climatic) processes in California since 9 Aug. 2017 and before 3 Mar. 2018 was already predicted (Simonenko, 2017). Based on the global prediction thermohydrogravidynamic principle used for the real planetary conf igurations of the Earth and the planets of the Solar System, it was calculated (Simonenko, 2017) the numerical time moment $\mathrm{t}=\left(\tau_{\mathrm{rr}}, 2 \mathrm{O} 17\right)=2017.85$ (related to the maximal in 2017 combined planetary and solar integral energy gravitational influence on the internal rigid core of the Earth). The time moment $t=\left(\tau_{c r}, 2017\right)=2017.85$ corresponds approximately to 7 Nov. 2017. Previously, based on the global prediction thermohydrogravidynamic principle used for the range $(2004,2016)$, it was calculated (Simonenko, 2016a, 2016b) the dates corresponding to the different local maximal combined planetary and solar integral energy gravitational influences on the internal rigid core of the Earth (for the real planetary conf igurations during the range 2004-2016). Simonenko, (2017) analyzed the previous signif icant earthquakes occurred in California near the calculated dates corresponding to the maximal combined planetary and solar integral energy gravitational influences on the Earth for 2004, 2005 , ..., 2016, correspondingly (as per U.S. Geological Survey). The simple approximate relations (Simonenko, 2017) are used for calculation of the dates $t_{c}$ (given in $\mathrm{yr}$ in the presented Table-1 below) of the considered dynamic (as per U.S. Geological Survey) earthquakes and for calculation of the corresponding differences $\triangle=\left|\mathrm{t}_{\mathrm{e}}-\mathrm{t}^{*}\left(\tau_{\mathrm{cr}}, 2017\right)\right|$ given in days in the presented Tables 1,2 below.

Considering the range (2004-2016) and analyzing the previous year-wise dynamic earthquakes occurred in California near the calculated dates corresponding to the maximal (in 2004 to 2016) combined planetary and solar integral energy gravitational influences on the internal rigid core of Earth, it was calculated (on 9 Aug. 2017). The different probabilities of the forthcoming strongest (in California) earthquakes (and related (Gutenberg, 1927; Simonenko, 2009, 2012, 2013, 2014a,b) strongest (in 2017 \& 2018) climatic processes) since 9 Aug. 2017 \& before 3 Mar. 2018) during the corresponding calculated time ranges (Simonenko, 2017).

Table-1 includes the previous (Simonenko, 2017) 
Table 1: Total dynamic earthquakes analysis occurred in California on dates $t_{\mathrm{e}}$ near the calculated date $\left.t^{*}\left(\tau_{\mathrm{cr}}, 2017\right)=2017.85\right)$

\begin{tabular}{|c|c|c|c|}
\hline Magnitude M, Region & Date $t_{e}$ of earthquake, in yr & Coordinate & $\triangle=\left|\mathrm{t}_{\mathrm{e}}-\mathrm{t}^{*}\left(\tau_{\mathrm{cr}}, 2017\right)\right|$ in days \\
\hline nia & $25,2018=$ & $6^{\circ} \mathrm{N}, 126.333^{\circ} \mathrm{W}$ & 79.78 days after the date ${ }^{@}$ \\
\hline $\mathrm{M}=4.0,12 \mathrm{~km} \mathrm{NE}$ of Trabuco Canyon, CA & January $25,2018=2018.068446$ & $33.741^{\circ} \mathrm{N}, 117.491^{\circ} \mathrm{W}$ & 79.78 days after the date ${ }^{@}$ \\
\hline $\mathrm{M}=3.9,8 \mathrm{~km}$ ENE of Alum Rock, CA & December 27, 2017=2017. 990587 & $37.397^{\circ} \mathrm{N}, 121.747^{\circ} \mathrm{W}$ & 51.34 days after the date ${ }^{@}$ \\
\hline $\mathrm{M}=4.0,14 \mathrm{~km} \mathrm{NE}$ of Julian, CA & December 7, 2017= 2017.9358309 & $33.148^{\circ} \mathrm{N}, 116.479^{\circ} \mathrm{W}$ & 31.34 days after the date ${ }^{@}$ \\
\hline $\mathrm{M}=4.6,22 \mathrm{~km}$ NE of Gonzales, California & November $13,2017=2017.868925$ & $36.631^{\circ} \mathrm{N}, 121.244^{\circ} \mathrm{W}$ & 6.91 days after the date ${ }^{@}$ \\
\hline $\mathrm{M}=3.6,5 \mathrm{~km} \mathrm{NW}$ of Westwood, CA & September $19,2017=2017 \cdot 71868$ & $34.087^{\circ} \mathrm{N}, 118.476^{\circ} \mathrm{W}$ & 47.96 days before the date ${ }^{\complement}$ \\
\hline
\end{tabular}

${ }^{\circledR} \mathrm{t}^{*}\left(\tau_{\mathrm{cr}}, 2017\right)$

partial combined analysis for 2017 (from Aug. 9, 2017 to Oct. 22, 2017) and the new additional analysis (from Nov. 27, 2017 to Mar. 3, 2018) of the dynamic earthquakes occurred in California on dates $t_{e}$ near the calculated date $t^{*}\left(\tau_{\mathrm{rr}}\right.$, 2017) $=2017.85$ (as per U.S. Geological Survey) corresponding to the maximal (in 2017) combined planetary and solar integral energy gravitational influence on the internal rigid core of the Earth. Considering (on Nov. 27, 2017 based on the part of Table 1 below Nov. 13, 2017) the dynamic earthquakes in California (as per U.S. Geological Survey) from Aug. 9, 2017 to Nov. 27, 2017, it was obvious that the date (Nov. 13, 2017) of the 4.6-magnitude earthquake (which struck $22 \mathrm{~km}$ NE of Gonzales, California near 6.91 days after the date $\left.t^{*}\left(\tau_{\mathrm{cr}}, 2017\right)=2017.85\right)$ belongs to the predicted range (9 Aug. $\div 29$ Nov., 2017) characterized by the probability $\mathrm{Pr}=0.6923$ of the strongest (in California for 2017) earthquakes and related (Gutenberg, 1927; Simonenko, 2009, 2012, 2013, 2014a,b) strongest climatic processes near the calculated numerical time moment. The obtained minimal difference of 6.91 days (Table-1) demonstrated the practical applicability of the global prediction thermohydrogravidynamic principle (Simonenko, 2012, 2014b) for the reasonable prediction of the seismotectonic activity in California for 2017 near the calculated (Simonenko, 2007) date of the maximal (in 2017) combined planetary and solar integral energy gravitational influence on the internal rigid Earth core.

Considering the devastation from California's most destructive wildfire it is evident, the convincing evidence of the practical applicability of the global prediction thermohydrogravidynamic principle for the reasonable prediction (Simonenko, 2017) of the strongest intensifications of the closely related seismotectonic and climatic processes in California (Gutenberg, 1927; Simonenko, 2009, 2012, 2013, 2014a, 2014b) determined by the maximal (in 2017 near the calculated date $\mathrm{t}^{*}\left(\tau_{\mathrm{r} r}\right.$, 2017 $=2017.85$ ) combined planetary and solar integral energy gravitational influence on the internal rigid Earth core. Considering (on Mar. 23, 2019 based on Table-1) the additional (from Oct 22, 2017 to Mar. 3, 2018) dynamic earthquakes in California, it is obvious that the date (Dec. 7 ,
2017) of the 4.o-magnitude earthquake (which struck 14 $\mathrm{km}$ NE of Julian, CA near 31.34 days after the date $\mathrm{t}^{*}\left(\tau_{\mathrm{cr}}\right.$, 2017 $=2017.85$ ) belongs to the predicted (Simonenko, 2017) range (9 Aug. $\div 8$ Dec., 2017) characterized by the probability $\mathrm{Pr}=0.7692$ of the strongest (in California for 2017) earthquakes and related strongest climatic processes near the numerical time moment $\mathrm{t}^{*}\left(\tau_{\mathrm{cr}}, 2017\right)=2017.85$. It is the first additional unquestionable fact confirming the total validity of the global prediction thermohydrogravidynamic principle concerning the strongest intensifications of the seismotectonic processes in California since 9 August, 2017 and before 3 Mar. 2018 (Simonenko, 2017). It is obvious (on Mar., 23, 2019; Table-1) that the date (Dec., 27, 2017) of the 3.9-magnitude earthquake (which struck $8 \mathrm{~km}$ ENE of Alum Rock, CA near 51.34 days after the date $\left.t^{*}\left(\tau_{\mathrm{cr}}, 2017\right)=2017.85\right)$ belongs to the predicted (Simonenko, 2017) range (9 Aug. $2017 \div 9$ Jan. 2018) characterized by the probability $\operatorname{Pr}=0.8461$ of the strongest (in California for 2017 \& 2018) earthquakes and related strongest climatic processes near the numerical time moment $\mathrm{t}^{*}\left(\tau_{\mathrm{cr}}, 2017\right)=2017.85$. It is obvious (based on Table 1) that the date (Jan. 4, 2018) of the 4.4-magnitude earthquake (which struck $2 \mathrm{~km}$ SE of Berkeley, CA near 58.78 days after the date $\left.t^{*}\left(\tau_{\mathrm{cr}}, 2017\right)=2017.85\right)$ belongs to the predicted (Simonenko, 2017) range (9 Aug. $2017 \div 9$ Jan. 2018) characterized by the probability $\operatorname{Pr}=0.8461$. It is obvious (based on Table-1) that the date (Jan. 25, 2018) of the 4.0-magnitude and 5.8-magnitude (strongest in California for 2017 and 2018 as per U.S. Geological Survey) earthquakes (which struck $12 \mathrm{~km}$ NE of Trabuco Canyon, CA and $175 \mathrm{~km}$ W of Ferndale, California, respectively, near 58.78 days after the date $\left.t^{*}\left(\tau_{c r}, 2017\right)=2017.85\right)$ belongs to the predicted (Simonenko, 2017) range (9 Aug. $2017 \div 3$ Mar. 2018) characterized by the probability $\operatorname{Pr}=0.99$ of the strongest (in California for 2017 \& 2018) earthquakes and related strongest climatic processes near the numerical time moment $\mathrm{t}^{*}\left(\tau_{\mathrm{cr}}, 2017\right)=2017.85$. The considered second, third, fourth and fifth additional unquestionable facts confirm the total validity of the global prediction $\tau \eta \varepsilon \rho \mu о \eta \psi \delta \rho о \gamma \rho \alpha \varpi \iota \delta \psi \nu \alpha \mu \iota \chi$ principle concerning the strongest intensifications of the seismotectonic processes 
Table 2: Total dynamic earthquakes analysis (characterized by the magnitude $\mathrm{M} \geq 7 \cdot 5$ ) occurred in California since 1900 and before 28 April, 2019

\begin{tabular}{llll}
\hline Magnitude M, Region & Date $\mathrm{t}_{\mathrm{e}}$ of earthquake, in yr & Coordinate & $\triangle=\left|\mathrm{t}_{\mathrm{e}}-\mathrm{t}^{*}\left(\tau_{\mathrm{cr}}, 1906 / 1952\right)\right|$ in days \\
\hline $\mathrm{M}=7.9$, San Pablo Bay, California & April 18, 1906 $=1906.299281$ & $37.750^{\circ} \mathrm{N}, 122.550^{\circ} \mathrm{W}$ & 36.78 days before the date $\mathrm{t}^{*}\left(\tau_{\mathrm{cr}}, 1906\right)$ \\
$\mathrm{M=7.5}$, 6 km WNW of Grapevine, CA & July 21, 1952 = 1952.557494 & $34.958^{\circ} \mathrm{N}, 118.998^{\circ} \mathrm{W}$ & 87.96 days after the date $\mathrm{t}^{*}\left(\tau_{\mathrm{cr}}, 1952\right)$
\end{tabular}

in California since 9 Aug., 2017 and before 3 Mar., 2018 (Simonenko, 2017).

To demonstrate additionally the validity of the global prediction thermohydrogravidynamic principle concerning the strongest intensifications of the seismotectonic processes in California, let us consider the strongest earthquakes (characterized by the magnitude as per U.S. Geological Survey) in California since 1900 and before 28 Apr. 2019. Table-2 includes the analysis of the strongest earthquakes (characterized by the magnitude $\mathrm{M} \geq 7.5$ as per U.S. Geological Survey) occurred in California since 1900 \& before 28 Apr. 2019.

The strongest earthquakes in California were realized on dates $t_{e}$ near the calculated dates $t^{*}\left(\tau_{c r}, 1906\right)=1906.4$ and $\mathrm{t}^{*}\left(\tau_{\mathrm{cr}}, 1952\right)=1952.31666666$ corresponding to the maximal combined planetary and solar integral energy gravitational influences on the internal rigid core of the Earth during 1906 \& 1952, respectively. These facts conf irm additionally the validity of the global prediction thermohydrogravidynamic principle explaining the strongest intensifications of the seismotectonic processes in California on April 18, 1906 and on July 21, 1952.

\section{Conclusion:}

In this update, the established generalized differential formulation of the first law of thermodynamics and the related established (Simonenko, 2012, 2014b) global prediction on thermohydrogravidynamic principles determining the maximal temporal intensifications of the global and regional natural (seismotectonic, volcanic, climatic and magnetic) processes of the Earth has already discussed (Simonenko, 2007, 2012, 2014a,b). The final analysis of the prediction (Simonenko, 2017) made on August 9, 2017 (based on the global prediction thermohydrogravidynamic principle taken into account the non-stationary gravitational field of the planets and the Sun) has also discussed. The prediction (Simonenko, 2017) was based also on the analysis of the previous dynamic (as per U.S. Geological Survey) earthquakes occurred since 2004 and before 2016 in California. It was proved the confirmed total validity of the prediction (Simonenko, 2017) concerning the strongest intensifications of the seismotectonic processes in California (since 9 Aug. 2017 \& before 3 Mar. 2018) determined by the maximal (near 7 Nov. 2017) combined planetary and solar integral energy gravitational influence on the internal rigid core of the Earth. It was shown in Table-1, the unquestionable fact that the date
(Jan. 25, 2018) of the (strongest in California for 2017 and 2018 as per U.S. Geological Survey) 5.8-magnitude earthquake belongs to the predicted (Simonenko, 2017) range (9 Aug. $2017 \div 3$ Mar. 2018) characterized by the probability $\mathrm{Pr}=0.99$ of the strongest (in California for 2017 \& 2018) earthquakes and related strongest climatic processes. The consideration of the strongest earthquakes (characterized by the magnitudes $\mathrm{M} \geq 7.5$ as per U.S. Geological Survey) in California (since 1900 \& before 28 Apr. 2019) has conf irmed additionally the validity of the global prediction thermohydrogravidynamic principle. The principle has explained (see Table-2) the strongest intensifications of the seismotectonic processes in California on Apr. 18, 1906 \& on Jul. 21, 1952. It was shown that the application of the global prediction thermohydrogravidynamic principle is the scientific way to predict (in advance) the modern strongest intensifications of the seismotectonic and climatic processes in California subjected to the energetic cosmic (planetary \& solar) gravitational influences.

\section{References:}

Alboussière, T., Deguen, R. \& Melzani, M. (2010): Meltinginduced stratification above the Earth's inner core due to convective translation. Nature, 466:744-747.

deGroot, S.R. \& Mazur, P. (1962): Non-equilibrium Thermodynamics. Pub. by: North-Holland and Publishing Company. P. 510.

Gibbs, J.W. (1873): Graphical methods in the thermodynamics of fluids. Pub. by: Connecticut Academy. P. 342.

Gutenberg, B. (1927): Grundlagen der Erdbebenkunde. Pub. by: Gebrüder Bornträger, Berlin, Germany. P. 189.

Gyarmati, I. (1970): Non-equilibrium Thermodynamics, Field Theory and Variational Principles. Pub. by: SpringerVerlag, Berlin, Germany. P. 184.

Landau, L.D. \& Lifshitz, E.M. (1976): Theoretical Physics, Vol. 5. Statistical Physics. Pub. by: Nauka, Moscow, Russia, In Russian, $\mathrm{p} 584$.

Richter, C.F. (1958): Elementary Seismology. Pub. by: W.H. Freeman, San Francisco, USA. P. 768.

Simonenko, S.V. (2004): The macroscopic non-equilibrium kinetic energies of a small fluid particle. L. Non-Equilib. Thermodyn., 29(1):107-123.

Simonenko, S.V. (2006): Non-equilibrium Statistical Thermohydrodynamics of Turbulence. Pub. by : Nauka, Moscow, Russia, p 174.

Simonenko, S.V. (2007): Thermohydrogravidynamics of the Solar System. Pub. by: Institute of Technology and Business Press, Nakhodka, Russia. P. 182. 
Simonenko, S.V. (2009): Fundamentals of the thermohydrogravidynamic Theory of Cosmic Genesis of the Planetary Cataclysms. Pub. by: Institute of Technology and Business Press, Nakhodka, Russia. P. 273.

Simonenko, S.V. (2012): The Cosmic Energy Gravitational Genesis of the Increase of the Seismic and Volcanic Activity of the Earth in the Beginning of the 21st Century AD. Pub. by: Institute of Technology and Business Press, Nakhodka, Russia. P. 220.

Simonenko, S.V. (2013): Fundamentals of the thermohydrogravidynamic theory of the global seismotectonic activity of the Earth. Int. J. Geophy., Article ID 519829, p. 39 .

Simonenko, S.V. (2014a): The practical forecasting aspects of the thermohydrogravidynamic theory of the global seismotectonic activity of the Earth concerning to the Japanese earthquakes near the Tokyo region. Am. J. Earth Sci., 1(1):38-61.

Simonenko S.V. (2014b): The prognosticating aspects of the developed cosmic geophysics concerning the subsequent forthcoming intensifications of the global seismicity, volcanic and climatic activity of the Earth in the 21st century. Br. J.Appl.Sci. Technol., 4(25):3563-3630.
Simonenko, S.V. (2015): The cosmic energy gravitational genesis of the forthcoming intensifications of the global seismotectonic, volcanic, climatic and magnetic activities since 2016 AD. Am. J. Earth Sci., 2(6):211-229.

Simonenko, S.V. (2016a): The prognosticating results of the cosmic seismology concerning the forthcoming intensification of the global seismotectonic, volcanic and climatic activities of the Earth from 1 September to 10 November, 2016AD. L.Adv. Environ. Sci., 1(1):90-101.

Simonenko, S.V. (2016b): The confirmed validity of the thermohydrogravidynamic theory concerning the strongest intensifications of the global natural processes of the Earth in 2016 since 1 September, 2016. Br. J. Appl. Sci. Technol., 18(5):1-20.

Simonenko, S.V. (2017): The prediction of the thermohydrogravidynamic theory concerning the strongest intensif ications of the seismotectonic and climatic processes in California since 9 August, 2017 and before 3 March, 2018. Int.J. Res. - Granthaalayah, 5(15):137-159. 